\title{
Development and Simulation of Adaptive Traffic Light Controller Using Artificial Bee Colony Algorithm
}

\author{
Risikat Folashade Adebiyi \\ Ahmadu Bello University/Department of Communication Engineering, Zaria, 234, Nigeria \\ E-mail: rfadebiyi@gmail.com \\ Kabir Ahmad Abubilal \\ Ahmadu Bello University/Department of Communication Engineering, Zaria, 234, Nigeria \\ E-mail: kabirahmed@abu.edu.ng \\ Muhammad Bashir Mu'azu \\ Ahmadu Bello University/Department of Computer Engineering, Zaria, 234, Nigeria \\ Email: mbmuazu@abu.edu.ng \\ Busayo Hadir Adebiyi \\ Ahmadu Bello University/Department of Computer Engineering, Zaria, 234, Nigeria \\ Email: busayoadebiyi@yahoo.com
}

Received: 29 May 2017; Accepted: 20 December 2017; Published: 08 August 2018

\begin{abstract}
This paper proposes an adaptive traffic control system that dynamically manages traffic phases and durations at cross-intersection. The developed model optimally schedules green light timing in accordance with traffic condition on each lane in order to minimize the Average Waiting Time (AWT) at the cross intersection. A MATLAB based Graphic User Interface (GUI) traffic control simulator was developed. Three scenarios of vehicular traffic control were simulated and the results presented. The results show that scenario one and two demonstrated the variation of the AWT and Performance of the developed algorithm with changes in the maximum allowable green light timing over the simulation interval. In the third scenario, an AWT of $38 \mathrm{sec}$ was recorded against a maximum allowable green light duration of $120 \mathrm{sec}$, during which 1382 vehicles were evacuated from the intersection, leaving 22 vehicles behind. The algorithm also had a performance of $98.43 \%$ over a simulation duration of $1800 \mathrm{sec}$.
\end{abstract}

Index Terms-Average Waiting Time, Artificial Bee colony, Queue Length, Graphic User Interface (GUI) and Congestion.

\section{INTRODUCTION}

Maintaining an efficient transportation system has been the main concern for public authorities cities confronted with multiple traffic jams each day, which cause large parts of it to become irresponsive to traffic movement(s) [1]. In a conventional traffic light controller, the traffic lights change at constant cycle times which are clearly not optimal [2]. It would be more feasible and sensible to pass more vehicles at the green time interval if there are fewer vehicles waiting behind the red lights or vice versa. However, general traffic situation may vary according to traffic demand, incidents or work construction. In order to overcome the fluctuations in traffic demand, a real-time traffic control is required. Since an intelligent traffic signal system is a major part of an intelligent transportation system, it is a challenge to increase the work efficiency of these traffic signals in order to reduce traffic jams and congestion [3]. We applied an Artificial Bee Colony based Adaptive Dynamic Scheduling Algorithm in the traffic control system to provide intelligent green time interval responses based on dynamic traffic load inputs. Thereby overcoming the inefficiencies of the conventional traffic controllers. The features inherent in Artificial Bee Colony that play a critical role in making it the best choice for traffic control management and analysis is the ability to aid easy modification by introducing model parameters that result in better performance which makes use of fewer parameters and has a good convergence. The system proposed in this paper is that the vehicles are detected using wireless sensor detectors with the information about each vehicle on the lane transmitted from the Reduced Function Devices (RFDs) to the Full Function Devices (FFDs). The FFDs then polls the information to the First Pan Coordinators (FPC) or base station.

This paper is organized as follows: Section I is an 
introduction to the paper discusses traffic congestion. Section II is a literature review about the relevant related research work that has been undertaken. Section III consist of the proposed methodology while Section IV discusses the results. Then, Section V concludes the work and consideration necessary for future works.

\section{RELATED WORKS}

This section gives a brief review of various approaches that have been employed to solve problems associated with adaptive traffic control using algorithms.

In the work of [4], a model based on image mosaicking technique was designed. The algorithm adopted the use of inter-arrival and inter-departure times to simulate the leaving of vehicles. The model provided good and satisfactory results of the Average Waiting Time.

A distributed algorithm for adaptive traffic light control which decides in a dynamic fashion, the green light sequences by selecting movements comprising of each phase and its duration was developed by [5]. The system model made use of sensors organized into four hierarchical layers with several communication paths. To ensure that the proposed method is plausible, simulations were conducted with positive results for the minimization of the average waiting time.

Application of fuzzy logic for multi-agent based autonomous traffic lights control system using wireless sensor networks was described by [6]. Real-time parameters such as traffic density and queue length were obtained by using image-processing techniques for the two junctions. The system was designed to consider emergency vehicles (ambulance, police unit, and fire brigade) coming from three different directions at the same time with different speed ratio. In the case of timevarying traffic, the proposed Fuzzy Logic System Control (FLSC) was superior to the fixed time controller. In the work of [7], an algorithm for vehicle queuing system using the Monte Carlo simulation technique was developed to reduce vehicle waiting time and vehicle queues at intersections in an urban region. The design of an adaptive traffic light controller using fuzzy logic Sugeno method was used to determine the green time interval at an intersection [8]. Three inputs namely the number of queues, waiting for time and traffic flow of vehicles were determined using simulation. Results showed that the traffic light using fuzzy logic control performed better than the fixed time control.

Management of an intelligent traffic light using the genetic algorithm that detected the level of congestion and the abnormal situations in two main highways and four intersections was proposed by [3]. The collection of data was achieved using a video imaging system. A realtime decision that determined the interval of green light time for each traffic light at each intersection was achieved. It was evident that the algorithm counted vehicles accurately with $96 \%$ performance.

An advanced traffic light system based on congestion estimation using fuzzy logic was presented [9]. The scenario of an isolated traffic junction was simulated based on congestion estimation. The results showed that the advanced traffic light control system outperformed the fixed traffic light control and the even the actuated traffic controllers. The evaluation of an adaptive traffic light control system in Istanbul, Turkey was introduced by [10]. They used a combination of the fuzzy and genetic algorithm as the optimization tool. Optimum signal timings were determined according to measured real-time traffic and occupancy. The performance had a $10 \%$ improvement in terms of cycle time and $15 \%$ for travel time.

It is evident that many researchers have made an effort to improve the efficiency of traffic signals using many intelligent systems evaluated using unrealistic traffic models which often include single intersection and static traffic volumes. The proposed model for this work introduced a more realistic control strategy through the development of an adaptive dynamic scheduling algorithm based on an artificial bee colony that is capable of controlling the vehicular traffic at road intersections.

\section{PRoposed MethoD}

The Artificial Bee Colony (ABC) algorithm is a population-based meta-heuristic optimization which is inspired by the foraging behaviour of honeybee swarm. The $\mathrm{ABC}$ colony is divided into employed bees and onlooker bee. In ABC algorithm, the position of a food source represents a possible solution to the optimization problem and the nectar amount of a food source corresponds to the quality (fitness) of the associated solution [11]. It has been established that $\mathrm{ABC}$ algorithm can be employed to determine the appropriate green light duration for each of the roads forming the intersection [12]. Hence, a new structure of traffic control system was described using Artificial Bee Colony based Adaptive Dynamic Scheduling Algorithm. The methodology used a Vehicular Traffic Control System model in order to ease implementation of traffic scenarios at a cross intersection.

In our proposed algorithm, the following assumptions were made:

1. Each of the road forming the intersection has two lanes that are wide enough to support three separate vehicle queue

2. Vehicles are allowed to queue-up on each lane according to their intended motion direction decision, i.e.

a) Left decision $\rightarrow$ Left part of the lane

b) Right decision $\rightarrow$ right part of the lane

c) Straight decision $\rightarrow$ center of the lane

3. Delay is not caused by service vehicles crossing the intersection in a non-conflicting manner i.e. vehicles do not maneuver their way while at the intersection.

4. The sensors can detect vehicles on each of the 
lanes regardless of their speed and position i.e. vehicles are accurately counted.

Using a VTCS equation for an objective function that has been minimized at any instant in time

$$
N=\sum_{i=1}^{n} N_{o i}+\lambda_{i} t-\mu_{i} t
$$

Where, $\mathrm{N}=$ Queue length

$\mathrm{N}_{\mathrm{oi}}=$ Initial Queue length

$\lambda_{\mathrm{i}}=$ Initial Arrival rate

$\mu_{\mathrm{i}}=$ Initial Departure rate

The ABC computes the fitness of a candidate solution $x_{i}$ using (2) [13].

$$
\text { fitness }\left(x_{i}\right)=\left\{\begin{array}{l}
\frac{1}{1+f\left(x_{i}\right)} \text { if } f\left(x_{i}\right) \geq 0 \\
1+\left|f\left(x_{i}\right)\right| \quad \text { otherwise }
\end{array}\right.
$$

For the problem of function optimization, where, $f$ is the function to be minimized.

The conventional objective function of $\mathrm{ABC}$ algorithm shown in equation (2) which best suites minimization problem. Meaning that the objective function will have the maximum value of unity. For values of $f\left(x_{1}\right)<0$, the objective function becomes greater than zero. This case is rare since the populations are usually set of random values ranging from zero and one.

Thus,

$$
\begin{gathered}
F_{o b j}=N\left(t_{g}\right) \\
F_{o b j}=\frac{1}{1+f\left(x_{1}\right)}
\end{gathered}
$$

where $t_{\mathrm{g}}=$ green light duration

$\mathrm{N}=$ queue length

$\mathrm{F}_{\mathrm{obj}}=$ Objective Function of ABC

By considering the average arrival rate, $\lambda$ and the average departure rate, $\mu$ of the intersection

The green light timing $t_{\mathrm{g}}$, the minimized equation in (2) yields:

$$
f\left(x_{i}\right)=\frac{1-\sum_{i=1}^{N}\left(N_{O I}+\lambda_{i} t_{g}-\mu t_{g}\right)}{\sum_{i=1}^{n}\left(N_{O I}+\lambda_{i} t_{g}-\mu t_{g}\right)}
$$

In this work, $t_{g \text { max }}$ limits the value of $t_{g}$. Let $t_{g}$ be a function of the population of bees $\left(x_{i}\right)$ and

$$
k=\sum_{i=1}^{m}\left(\lambda_{i}-\mu_{i}\right)
$$

Therefore:

$$
t_{g}=t_{g \max } g\left(x_{i}\right)
$$

Where,

$$
\mathrm{g}\left(\mathrm{x}_{\mathrm{i}}\right)=\text { is a function of }\left(\mathrm{x}_{\mathrm{i}}\right)
$$

Since $x_{i}$ represent the $\mathrm{i}^{\text {th }}$ bee, $x_{i}$ is a row of number of parameters $(n p)$ which is a set of random real numbers taken in the range $(0,1), t_{g}$ can be redefined as follows.

Let:

$$
g\left(x_{i}\right)=\frac{\sum_{k=1}^{n p} x_{i}}{n p}
$$

By substituting $g\left(x_{i}\right), k$ into equation (5) yields;

The equation (6) represents the $\mathrm{ABC}$ based VTCS objective function.

$$
f\left(x_{i}\right)=\frac{n p-k t_{g \max } \sum_{k=1}^{n p} x_{i}}{k t_{g \max } \sum_{k=1}^{n p} x_{i}}
$$

The Adaptive dynamic Scheduling algorithm runs real-time traffic light regulation of phase sequence and green time duration based on data gathered by the Wireless Sensor Network (WSN) on the road [14]. The phase sequence is determined by using queue length for each input variable or flow or by sorting them in descending order of priority [15]. The phase sequence assigns priority to each phase equal to the maximum queue length of that phase. The calculation of green time is then considered.

The flowchart describing an Adaptive Dynamic Scheduling Algorithm based Vehicular Traffic Control System is shown in Fig. 1.

\section{RESULTS AND DISCUSSION}

The simulations use provisions of Average Arrival Rate (AAR), Average Departure Rate (ADR), ABC parameters and other parameters which includes Start time, green light timing, $t_{\text {gmax }}$ and simulation duration. The objective of these parameters is to observe the adaptive traffic light controller using Artificial Bee Colony based Adaptive Dynamic Scheduling Algorithm that minimizes AWT at cross-intersections. The 
developed MATLAB GUI is shown in the Fig. 2. The GUI aids in inputting various parameters required for simulations. In Table 1, Scenario one considers equal values of Average Arrival and Departure Rates. The maximum allowable green light duration is varied between 50 and 150 seconds and simulated over a duration of 300 seconds. The values for the percentage performance were noted and plotted against the maximum allowable green light duration as shown in Fig. 3. A vehicular traffic controller is designed with the aim of maintaining the average waiting time within a safe maximum. The lower the AWT, the better the controller. AWT may vary depending on the condition of the intersection at any given instant in time. Table 2, is intended to simulate a case in which the Average Arrival and Departure Rates are unequal across the cross-road for scenario two. It could be observed in Fig. 4, that the variation of Average Waiting Time (AWT) curve is less compared to that of Fig. 3. This may be associated with the pattern of the Average Arrival Rate (AAR). In scenario three shown in Table 3 generates and discusses all the features of the developed simulator. The maximum green light timing duration was kept at 2 minutes (120 seconds) and simulated over a duration of
30 minutes (1800 seconds). The simulation was initialized with $\mathrm{t}=60$ seconds, implying that vehicles were allowed to queue-up for 1 minute across the entire roads. Fig. 5 shows the variation of queue length with simulation time. It could be observed that a zero green light duration was generated by the controller at approximate point(s) 500, 900 and 1700 seconds which corresponds to a cleared condition. The ADSA is designed to generate a no green light $\left(\mathrm{T}_{\mathrm{gi}}\right)$ of zero whenever any road has no vehicle on it. This means that roads with no vehicles are skipped during simulation for improvement in performance. Fig. 6 illustrates the cumulative sum of all the vehicles evacuation from the intersection. The peak of the curve indicates that at the end of the simulation time of 30 minutes, almost 1400 vehicles were cleared from the intersection. The Fig. 7 can be observed to have almost exact form as Fig. 5. This is based on the fact that the AWT is fully controlled by the green lights scheduled for the individual roads forming the intersection. The AWT is highly sensitive to green light duration and queue length. Some of the important output parameters generated by the developed simulator were recorded at the end of Scenario 3. These parameters are presented in Table 4.

Table 1. Scenario One (Constant Arrival and Departure rates)

\begin{tabular}{|c|c|c|c|c|c|c|}
\hline \multicolumn{7}{|c|}{ Scenario One (Constant Arrival and Departure rates) } \\
\hline $\begin{array}{c}\text { Road } \\
\text { S/N }\end{array}$ & AAR & ADR & $\begin{array}{c}\text { ABC } \\
\text { Par. }\end{array}$ & Value & $\begin{array}{c}\text { Other } \\
\text { Parameters }\end{array}$ & Value \\
\hline 1 & $5 / 60$ & $5 / 60$ & Pop size & 10 & Start time & 0 \\
\hline 2 & $5 / 60$ & $5 / 60$ & Maxgen & 10 & $T_{\text {gmax }}$ & $50-150 \mathrm{~s}$ \\
\hline 3 & $5 / 60$ & $5 / 60$ & Np & 10 & $\begin{array}{c}\text { Simualtion } \\
\text { Duration }\end{array}$ & $300 \mathrm{~s}$ \\
\hline 4 & $5 / 60$ & $5 / 60$ & & & & \\
\hline
\end{tabular}

Table 2. Scenario Two (Variable Arrival and Departure rates)

\begin{tabular}{|c|c|c|c|c|c|c|}
\hline \multicolumn{7}{|c|}{ Scenario Two (Variable Arrival and Departure rates) } \\
\hline $\begin{array}{c}\text { Road } \\
\text { S/N }\end{array}$ & AAR & ADR & $\begin{array}{c}\text { ABC } \\
\text { Par. }\end{array}$ & Value & $\begin{array}{c}\text { Other } \\
\text { Parameters }\end{array}$ & Value \\
\hline 1 & $1(5 / 60)$ & $4(5 / 60)$ & Pop size & 10 & Start time & 0 \\
\hline 2 & $2(5 / 60)$ & $3(5 / 60)$ & Maxgen & 10 & $T_{\text {gmax }}$ & $50-150 \mathrm{~s}$ \\
\hline 3 & $3(5 / 60)$ & $2(5 / 60)$ & Np & 10 & $\begin{array}{c}\text { Simualtion } \\
\text { Duration }\end{array}$ & $300 \mathrm{~s}$ \\
\hline 4 & $4(5 / 60)$ & $1(5 / 60)$ & & & & \\
\hline
\end{tabular}

Table 3. Scenario Three (Arrival and Departure rates at 0.2 and 0.5 respectively)

\begin{tabular}{|c|c|c|c|c|c|c|}
\hline \multicolumn{7}{|c|}{ Scenario Three (Arrival and Departure rates at 0.2 and 0.5 respectively) } \\
\hline $\begin{array}{c}\text { Road } \\
\text { S/N }\end{array}$ & AAR & ADR & $\begin{array}{c}\text { ABC } \\
\text { Par. }\end{array}$ & Value & $\begin{array}{c}\text { Other } \\
\text { Parameters }\end{array}$ & Value \\
\hline 1 & 0.2 & 0.5 & Pop size & 10 & Start time & 0 \\
\hline 2 & 0.2 & 0.5 & Maxgen & 10 & $\mathrm{~T}_{\text {gmax }}$ & $120 \mathrm{~s}$ \\
\hline 3 & 0.2 & 0.5 & $\mathrm{~Np}$ & 10 & $\begin{array}{c}\text { Simualtion } \\
\text { Duration }\end{array}$ & $1800 \mathrm{~s}$ \\
\hline 4 & 0.2 & 0.5 & & & & \\
\hline
\end{tabular}




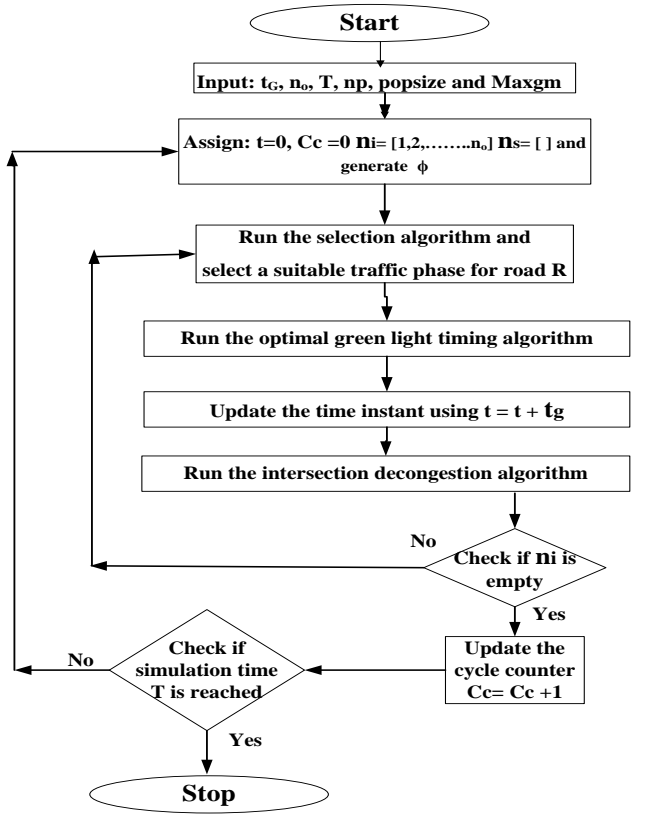

Fig.1. Flowchart of ADSA Algorithm for VTCS

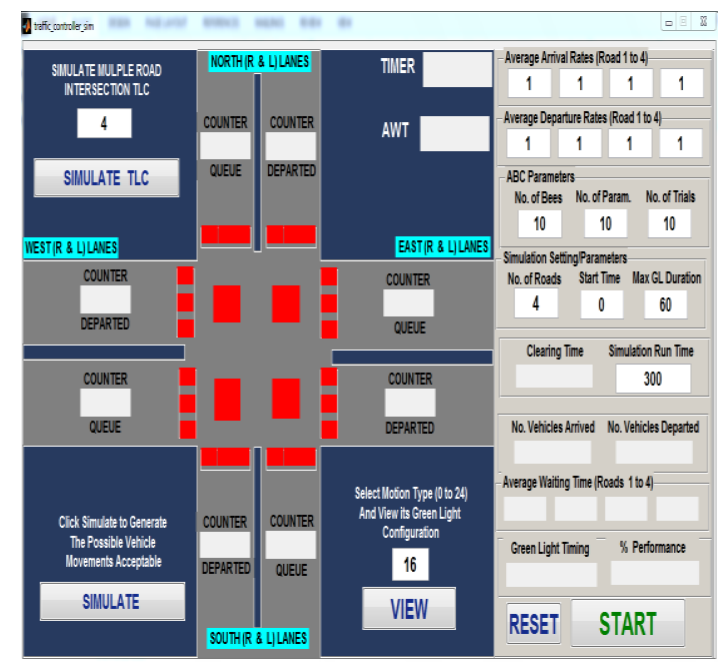

Fig.2. Graphic User Interface of the Developed Simulator

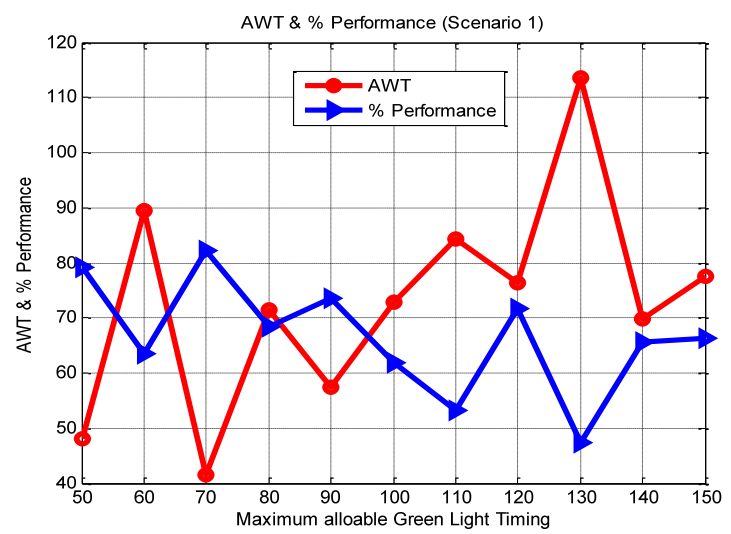

Fig.3. Variation of AWT and \% Performance with $\mathrm{T}_{\mathrm{gmax}}$ (Scenario one)

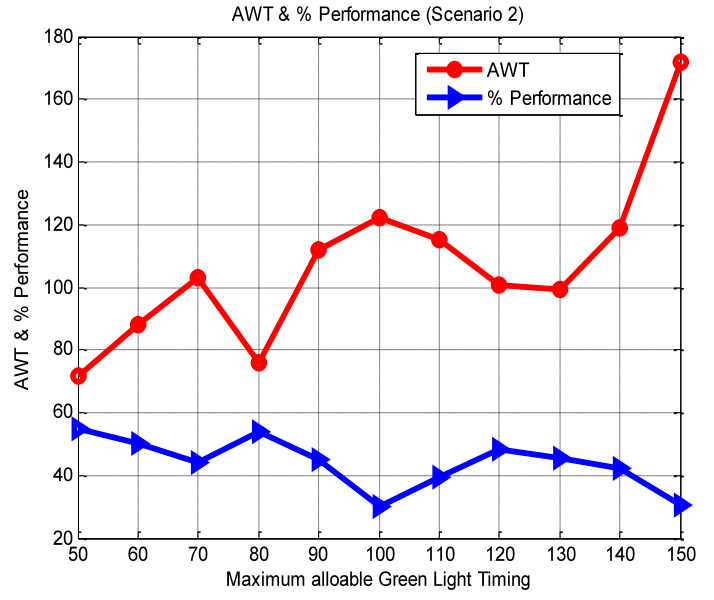

Fig.4. Variation of AWT and \% Performance with $\mathrm{T}_{\text {gmax }}$ (Scenario two)

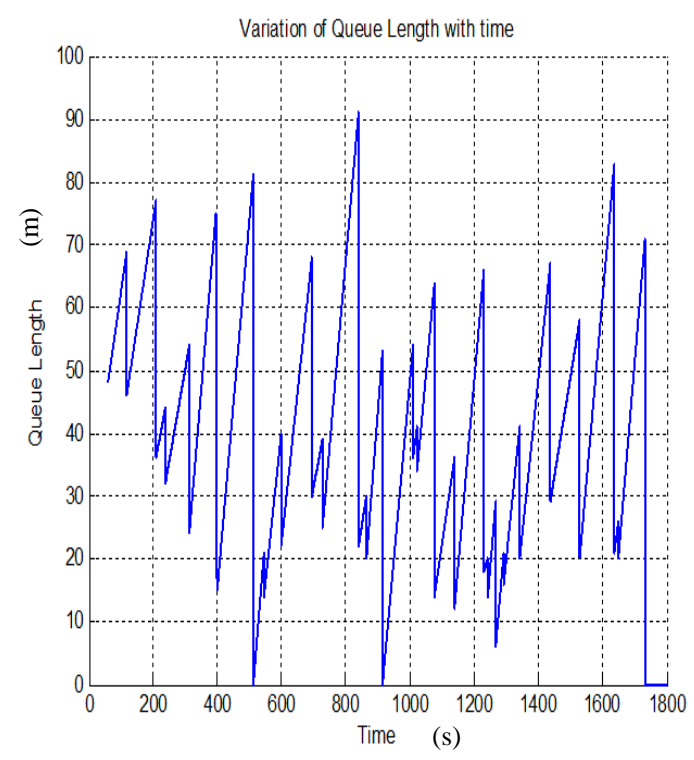

Fig.5. Variation of Queue Length with Time

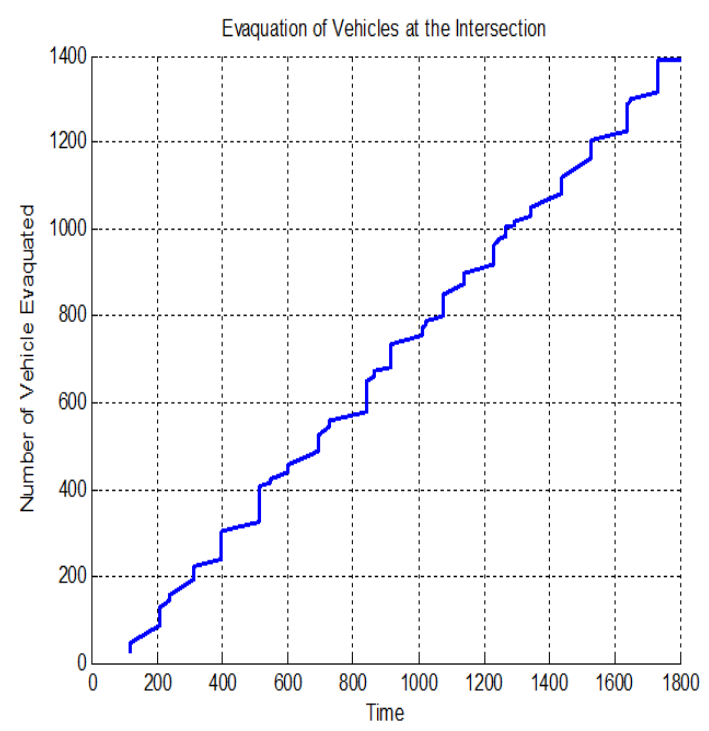

Fig.6. Evacuation of Vehicles at the Intersection 


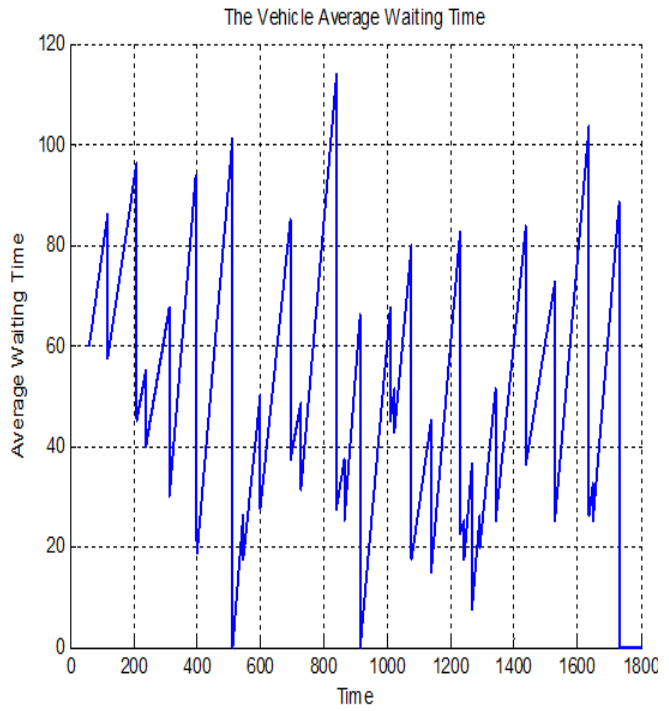

Fig.7. Vehicles Average Waiting Time

Table 4. Simulation Results for Scenario 3

\begin{tabular}{|c|c|}
\hline PARAMETER & VALUE \\
\hline $\mathrm{T}_{\mathrm{gmax}}$ & $120 \mathrm{~s}$ \\
\hline $\mathrm{AWT}$ & $38 \mathrm{~s}$ \\
\hline Clearing Time & $1640 \mathrm{~s}$ \\
\hline $\begin{array}{c}\text { Simulation Duration } \\
\text { Total Departure after } \\
1800 \mathrm{~s}\end{array}$ & $1800 \mathrm{~s}$ \\
\hline $\begin{array}{c}\text { Total Queue Length at } \\
\mathrm{t}=1800 \mathrm{~s}\end{array}$ & 22 vehicles. \\
\hline Performance & $98.43 \%$ \\
\hline Start Time & $60 \mathrm{~s}$ \\
\hline
\end{tabular}

\section{CONCLUSION}

In this paper, a proposed algorithm to control traffic light at cross intersections. The developed model optimally scheduled green light timing was done in accordance with traffic condition in order to minimize the Average Waiting Time (AWT) at the cross intersection. A MATLAB based Graphic User Interface (GUI) traffic control simulator was developed. Three scenarios of vehicular traffic control were simulated and results presented. The results showed satisfactory result in response to AWT at cross intersection.

In future work, the developed vehicular traffic control simulator should be replicated with java, while incorporating traffic motion controllers and compiled into real software that can run on various PCs in order to aid students in simulating any desired traffic control situation.

\section{REFERENCES}

[1] Cosariu, C., L. Prodan, and M. Vladutiu. Toward traffic movement optimization using adaptive inter-traffic signaling. in Computational Intelligence and Informatics (CINTI), 2013 IEEE 14th International Symposium on. 2013. IEEE.

[2] Subramaniam, S., M. Esro, and F. Aw, Self-Algorithm Traffic Light Controllers for Heavily Congested Urban
Route. WSEAS Transactions on Circuits and Systems, 2012. 11(4): p. 115-124.

[3] Odeh, S.M., Management of an intelligent traffic light system by using genetic algorithm. Journal of Image and Graphics, 2013. 1(2): p. 90-93.

[4] Mittal, P.K. and Y. Singh, Analysis and designing of proposed intelligent road traffic congestion control system with image mosaicking technique. International Journal of IT, Engineering and Applied Science Research (IJIEASR) Vol, 2013. 2: p. 27-31.

[5] Faye, S., C. Chaudet, and I. Demeure. A distributed algorithm for adaptive traffic lights control. in Intelligent Transportation Systems (ITSC), 2012 15th International IEEE Conference on. 2012. IEEE.

[6] Salehi, M., I. Sepahvand, and M. Yarahmadi, TLCSBFL: A Traffic Lights Control System Based on Fuzzy Logic. International Journal of u-and e-Service, Science and Technology, 2014. 7(3).

[7] Wang, F., et al., Simulation Analysis and Improvement of the Vehicle Queuing System on Intersections Based on MATLAB. Open Cybernetics \& Systemics Journal, 2014. 8: p. 217-223.

[8] Erwan, E.P., W. Oyas, and S. Selo, Design and Simulation of Adaptive Traffic Light Controller Using Fuzzy Logic Control Sugeno Method. International Journal of Scientific and Research Publications, 2015. 5(4): p. 6.

[9] Alam, J., Advance traffic light system based on congestion estimation using fuzzy logic. 2014.

[10] Gündoğan, F., et al., An Evaluation of Adaptive Traffic Control System in Istanbul, Turkey. Journal of Traffic and Logistics Engineering Vol, 2014. 2(3).

[11] Karaboga, D., et al., A comprehensive survey: artificial bee colony $(A B C)$ algorithm and applications. Artificial Intelligence Review, 2014. 42(1): p. 21-57.

[12] Adebiyi, R.F., et al., Management of Vehicular Traffic System Using Artificial Bee Colony Algorithm. International Journal of Images, Graphics and Signal Processing 2017. 9(11): p. 18-28.

[13] Priti Bansal, S.S. and Nitish Mittal, A Hybrid Artificial Bee Colony and Harmony Search Algorithm to Generate Covering Arrays for Pair-wise Testing. International Journal of Intelligent Systems and Applications (IJISA), 2017. 9(8): p. 59-70.

[14] Aljaafreh, A. and N. Al Oudat. Optimized Timing Parameters for Real-Time Adaptive Traffic Signal Controller. in Computer Modelling and Simulation (UKSim), 2014 UKSim-AMSS 16th International Conference on. 2014. IEEE.

[15] Pau, G. and G. Scata, Smart traffic light junction management using wireless sensor networks. WSEAS transactions on communication, 2014. 14: p. 2224-2864.

\section{Authors' Profiles}

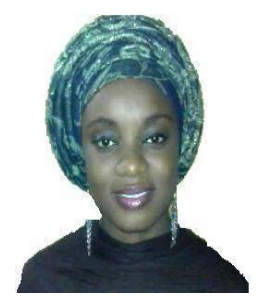

Risikat Folashade O. Adebiyi was born in Maiduguri, Borno State, Nigeria. She received B.Eng. degree in (Electrical and Electronics) engineering from University of Maiduguri, in 2011. She earned her M.Sc. degree in Telecommunications Engineering from Ahmadu Bello University Zaria, Nigeria in 2017 and presently pursuing her Ph.D in the department of Communication Engineering, of the same University. She is a 
Certified Fiber Optic Technician (CFOT) obtained from Etisalat Academy, Dubai in 2015. Her research interests include Wireless and mobile communications, Intelligent Transportation Systems and Artificial intelligence techniques.

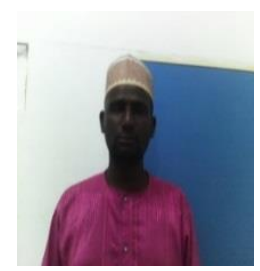

Kabir Ahmad Abubilal was born in Zaria, Kaduna State. He received his B.Eng. (Electrical Engineering) in 2006, M.Sc. (Electrical Engineering) in 2009 and Ph.D. (Electrical Engineering) in 2015 all from Ahmadu Bello University Zaria, Nigeria. He has several publications and conference proceedings. He won the best student paper award of the 2013 International Conference of Electrical and Electronics Engineering in World Congress of Engineering, London 2013. His research interests are Wireless and mobile communications, Microcontrollers and applications and Digital electronics.

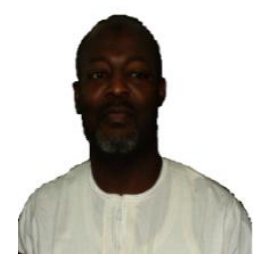

Muhammad Bashir Mu'azu is a Professor of Computational Intelligence. $\mathrm{He}$ obtained his B.Eng (Electrical Engineering) in 1991, MSc. (Electrical Engineering) in 2001 and Ph.D. (Electrical Engineering) with a specialization in Computational Intelligence in 2006 from Ahmadu Bello University Zaria, Nigeria. He has authored several refereed technical journal articles and conference proceedings. His research interests include; Control System Dynamics, Modeling and Optimization, Fuzzy Time Series Forecasting and Artificial Intelligence Optimization.

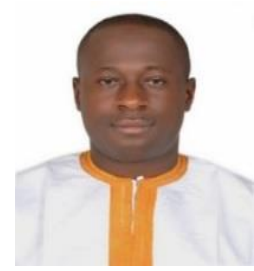

Techniques.

Busayo Hadir Adebiyi holds M.Sc and B.Sc (Hons) in Control Engineering and Physics respectively from Ahmadu Bello University, Zaria. He also holds Postgraduate Diploma in Electrical Engineering. His research interests include Control System Dynamics, Modelling and Optimization and Artificial Intelligence

How to cite this paper: Risikat Folashade Adebiyi, Kabir Ahmad Abubilal, Muhammad Bashir Mu'azu, Busayo Hadir Adebiyi, "Development and Simulation of Adaptive Traffic Light Controller Using Artificial Bee Colony Algorithm", International Journal of Intelligent Systems and Applications(IJISA), Vol.10, No.8, pp.68-74, 2018. DOI: 10.5815/ijisa.2018.08.06 